\title{
Rare and Unpredictable Cardiac Complication After Successful Elective Percutaneous Coronary Intervention: A Case Report
}

\author{
Roberto Ramos Barbosa ${ }^{1,2 *}$, Denis Moulin dos Reis Bayerl ${ }^{1}$, Ingrid Ardisson Colodete ${ }^{2}$, Carla Campos \\ Miranda $^{2}$, Mayara da Silva ${ }^{2}$ and Luiz Fernando Machado Barbosa ${ }^{1,2}$ \\ ${ }^{1}$ Unimed Vitória Hospital, Vitória, ES - Brazil \\ ${ }^{2}$ School of Medicine of Santa Casa de Misericórdia de Vitória, Vitória, ES - Brazil
}

*Corresponding author: Roberto Ramos Barbosa, Department of Cardiology, Unimed Vitória Hospital, School of Medicine of Santa Casa de Misericórdia de Vitória ES -Brazil.

Received Date: June 21, 2019

Published Date: June 27, 2019

\begin{abstract}
Left ventricular pseudoaneurysm is a rare yet potentially fatal complication observed usually in cases of ST-elevation myocardial infarction without timely reperfusion. We report a case of successful elective percutaneous coronary intervention that evolved into left ventricular apical pseudoaneurysm with pericardial effusion and cardiac tamponade, despite absence of reasonable cause for the complication, such as acute stent thrombosis or coronary perforation.
\end{abstract}

Keywords: Cardiac tamponade; Emergency; Left ventricular pseudoaneurysm; Percutaneous coronary intervention; Pericardial effusion

\section{Introduction}

Percutaneous coronary intervention (PCI) with stent implantation is a widely accepted approach for myocardial revascularization. It clearly reduces life-threatening complications associated with acute myocardial infarction (AMI) by providing effective reperfusion at an early onset when used in an acute setting $[1,2]$. However, one of the most feared complications of AMI is the formation of a left ventricular pseudoaneurysm. This serious cardiac entity occurs due to a contained rupture of the adherent pericardium wall which leads to thrombus and hematoma organization. Despite rare occurrence, left ventricular pseudoaneurysms have a high propensity to spontaneously rupture, frequently resulting in cardiac tamponade, obstructive shock and even sudden death [3].

Available literature reports incidence of left ventricular pseudoaneurysms post cardiac surgery as lower than $0.5 \%$ [4]. Its incidence in elective PCIs is unknown, mainly as it is unexpected unless severe ischemic myocardial injury occurs during the procedure. We report a challenging case of a successful elective PCI which evolved into left ventricular apical pseudoaneurysm and cardiac tamponade, a rare and dangerous condition of uncertain cause in this case.

\section{Case Report}

A 74-year-old dyslipidemic male was admitted to our tertiarycare centre for elective coronary angiography due to a diagnosis of stable angina and a treadmill test suggestive of myocardial ischemia. Earlier investigations from another tertiary-care centre revealed normal electrocardiogram and echocardiogram. The patient was physically active and presented with excellent cardiorespiratory condition. He was previously advised $20 \mathrm{mg}$ athorvastatin daily. However, after the onset of symptoms, $100 \mathrm{mg}$ aspirin daily was also advised. He had normal blood pressure and resting heart rate was 58 beats/min. Sinus rhythm was normal. Transradial coronary angiogram revealed $80 \%$ stenosis in the left circumflex (LCX) artery and $90 \%$ stenosis in proximal left anterior descending (LAD) artery (Figures 1A\&B).

Elective double-vessel percutaneous coronary intervention (PCI) was planned to manage the patient. The patient was hospitalized and $300 \mathrm{mg}$ clopidogrel daily was initiated which was then reduced to $75 \mathrm{mg}$ clopidogrel daily. On the second day, transfemoral PCI was successfully performed with the implantation of three Biomatrix Flex drug-eluting stents (DES) (Biosensors 
International, Singapore). Two DES were implanted in the LAD whilst one DES was implanted in LCX. Coronary blood flow and pressure were normal with no complaints of chest pain from the patient during the procedure. No intraprocedural complications occurred and the angiographic result was excellent (Figures 1C\&D). However, within the first 12 hours after the procedure, the patient complained of mild chest pain. Electrocardiographic abnormalities were not found.
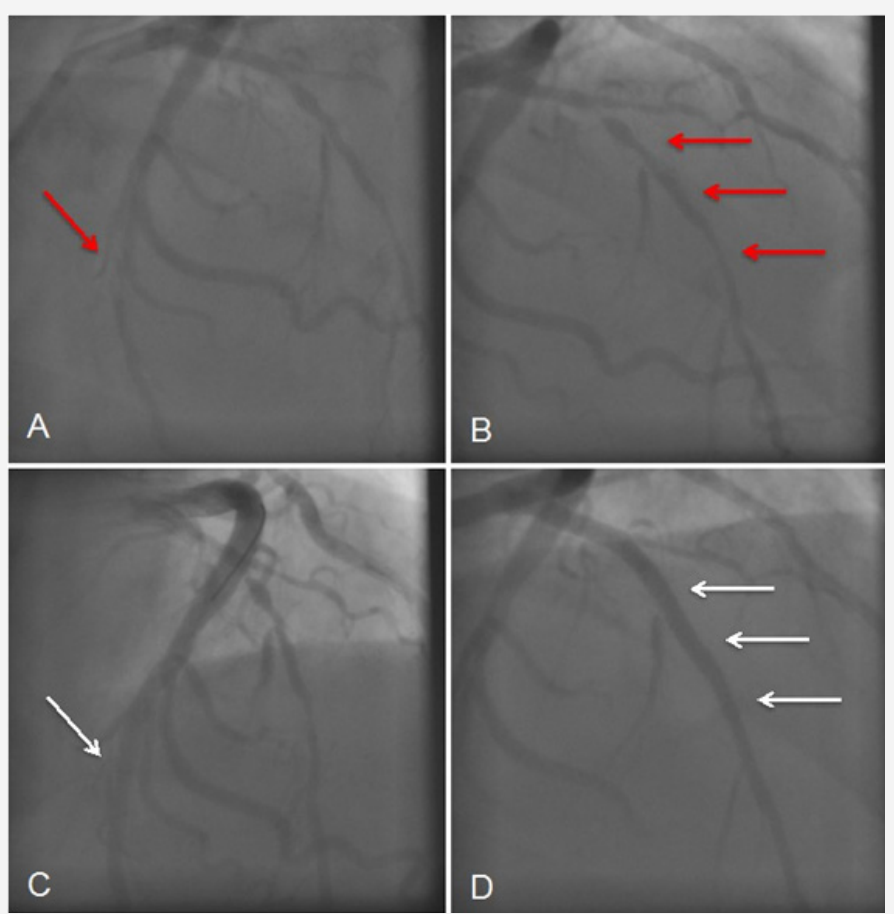

Figure 1: Coronary angiography before and immediately after elective percutaneous coronary intervention showing: (A) Severe stenosis $(80 \%)$ of left circumflex artery seen on coronary angiogram; (B) Severe and extent stenosis $(90 \%)$ of left anterior descending artery seen on coronary angiogram; (C) Angiographic result of left circumflex artery after percutaneous coronary intervention with implantation of one Biolimus-eluting stent; and (D) Angiographic result of left anterior descending artery after percutaneous coronary intervention with implantation of two Biolimus-eluting stents.

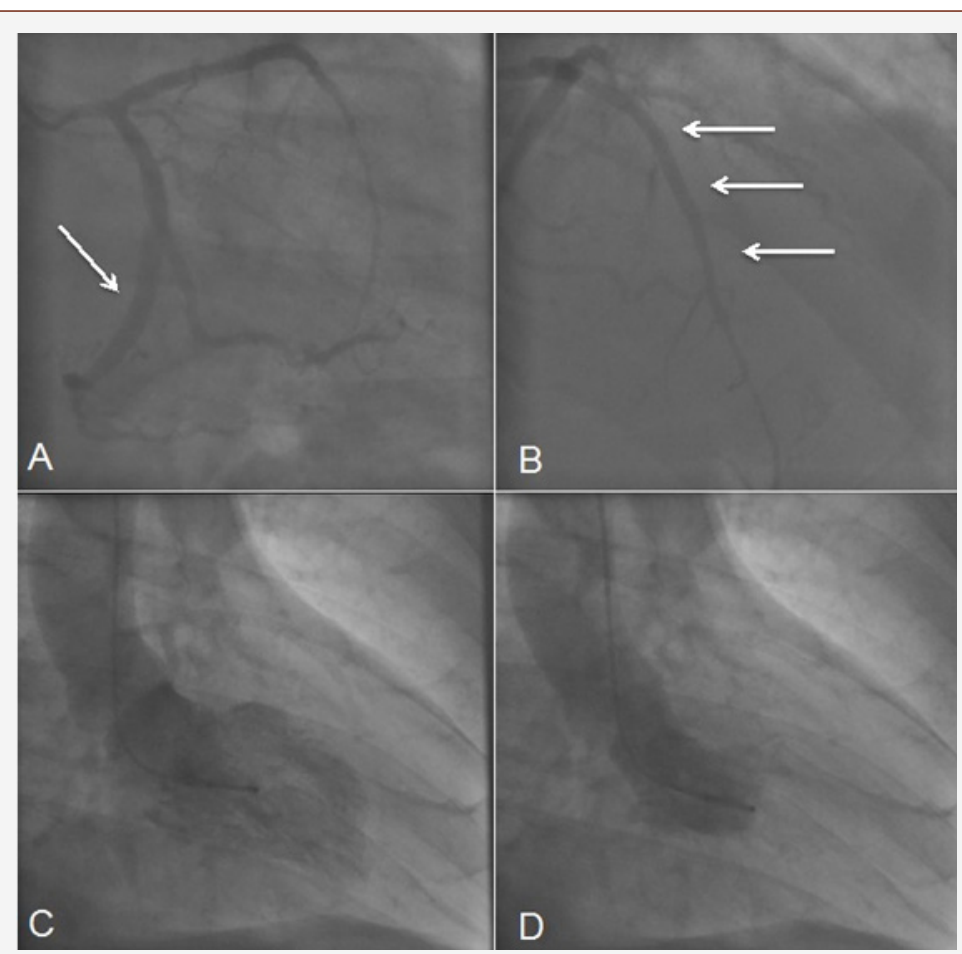

Figure 2: Urgent coronary angiography and left ventriculography performed 24 hours after elective percutaneous coronary intervention, facing the clinical hypothesis of acute occlusion of the left anterior descending artery showing: (A) Patent stent on the left circumflex artery with normal flow; (B) Patent stent on the left anterior descending artery with normal flow, with no signs of thrombi formation or coronary perforation. Mild coronary spasm was seen in distal left anterior descending artery; (C) Left ventriculogram (diastole) with no clear signs of apical pseudoaneurysm, chamber perforation or contrast extravasation to pericardium; and (D) Left ventriculogram (systole) with apparent normal contraction and no clear signs of apical pseudoaneurysm, chamber perforation or contrast extravasation to pericardium. 
A day after PCI was performed, the patient presented with worsening hypotension, sweating and chest pain. Transthoracic echocardiogram revealed left ventricular apical pseudoaneurysm and moderate pericardial effusion. Due to a likely diagnosis of periprocedural acute myocardial infarction caused by LAD acute stent thrombosis, urgent coronary angiography was performed which revealed patent stents with normal coronary blood flow and absence of intraluminal thrombi or extravasations due to coronary perforation (Figures 2A\&B). Only mild coronary spasm was seen in distal LAD. Left ventriculography showed no clear signs of perforation or contrast extravasation to pericardium (Figures 2C\&D). Close echocardiographic follow-up in the following hours displayed an image compatible with ruptured apical pseudoaneurysm, an increase in pericardial effusion and signs of cardiac tamponade with right chamber collapses (Figure 3).

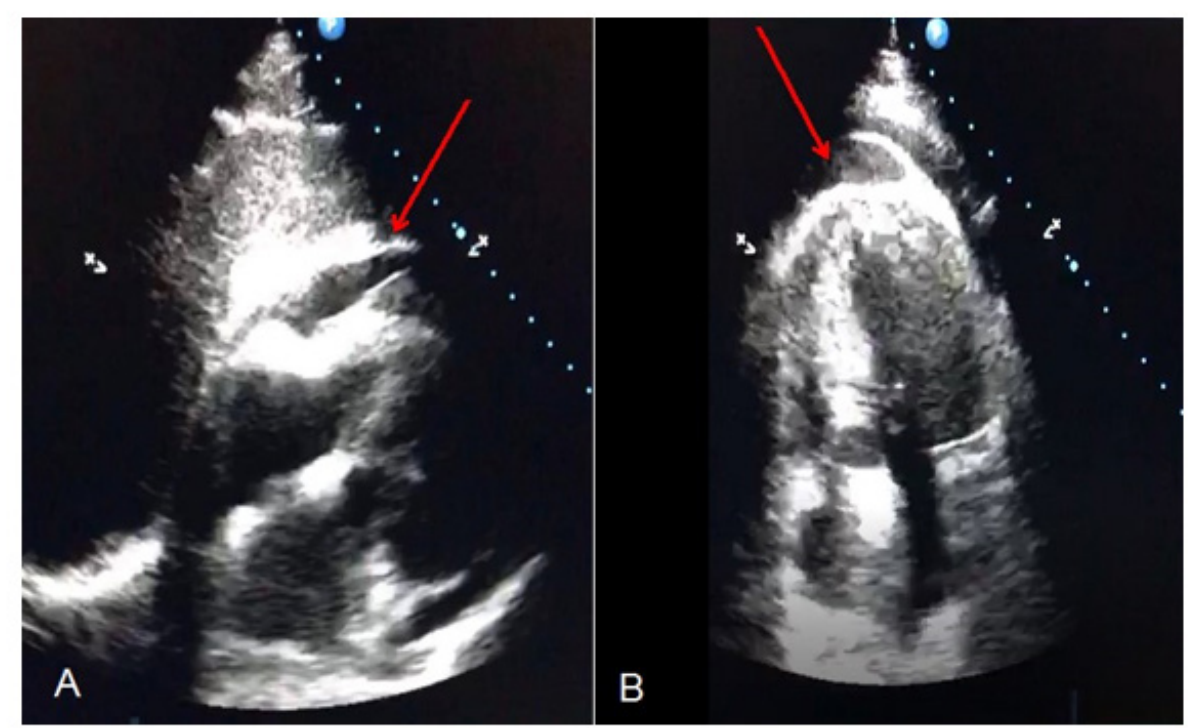

Figure 3: Echocardiography after 24 hours of eletive percutaneous coronary intervention showing: (A) Significant pericardial effusion with evidence of hemodynamic repercussion (right ventricular diastolic collapse); and (B) Pseudoaneurysm visualized on the left ventricle apex.

During the evening of the second day, i.e. 28 hours after PCI was performed, the patient underwent cardiac surgery with sternotomy for drainage of hemopericardium. A total volume of $800 \mathrm{~mL}$ was drained. A ruptured pseudoaneurysm at the left ventricular apex with friable and necrotic tissue were visualized. A local block with biological glue and hemostatic gelation sponge was successfully performed.

During the postoperative time period, the patient presented with acute kidney injury requiring hemodialysis, severe delirium and intravenous catheter-related infection. With intense and effective treatment, the patient recovered fully. He was discharged after 19 days with serum creatinine levels of $2.2 \mathrm{mg} / \mathrm{dL}$ and was able to resume regular physical activity. He remained asymptomatic at the 12-month clinical follow-up with normal renal and left ventricular functions. Informed consent was obtained from the patient prior to producing this case report.

\section{Discussion}

Facing an uncommon complication may be a difficult challenge in many medical situations. Whilst understanding the reasons for the unpredicted event, decision-making becomes an even greater struggle as timely interventions remain critical too. In this case report, we discuss the dilemmas related to the symptoms, rupture, diagnosis, and treatment of a left ventricular pseudoaneurysm.

Left ventricular pseudoaneurysms display a spectrum of clinical presentations. Symptoms may be non-specific including congestive heart failure, chest pain, and dyspnea or arrhythmia which result in a delayed diagnosis. These are also the most common symptoms. Other symptoms include sudden cardiac arrest, AMI, syncope, tamponade, and embolism. To-and fro murmurs are heard in two-thirds of patients. Patients may even present as completely asymptomatic [5]. Frances et al. [4] in their review reported 10\% asymptomatic patients, whilst Yeo et al. [6] reported as many as $48 \%$ asymptomatic patients.

The primary goal of treatment in ST-elevation myocardial infarction is re-establishment of arterial blood flow with reperfusion. However, reperfusion itself may injure the myocardium $[7,8]$. Such injury occurs after re-establishment of blood flow to the occluded coronary artery and hence distinct consequences include arrhythmias, left ventricular dysfunction, and microvascular occlusion. Furthermore, challenging obstructive coronary lesions may cause flow disturbances even in elective PCI in a stable coronary artery disease scenario.

There is a possibility that, in the case of an acute stent thrombosis, due to unforeseen reasons, intrinsic fibrinolytic mechanisms with the additional effect of standard antithrombotic drugs administrated could cause spontaneous recanalization. Cases of rapid healing after coronary dissection during PCI and spontaneous recanalizations during ST-elevation AMI have been reported [9-11]. Spontaneous reperfusion in the setting of STelevation acute coronary syndrome is reported in up to $30 \%$ of patients, with very few specific recommendations, although with favorable outcomes despite not undergoing immediate reperfusion [12]. 
In our patient, there was no flow disturbance during PCI to explain the extent of left ventricular damage due to myocardial ischemia. Prior to the new coronary angiogram, acute stent thrombosis and LAD occlusion was our main hypothesis despite no ST-elevation in the anterior wall. Thus, in our case, one possible explanation could be myocardial reperfusion injury after ballooncatheter expansion and stent deployment. The limited time of coronary occlusion during these procedural steps rarely results in serious damage. However, chest pain is frequently caused. Another possible reason for the complication is an acute stent thrombosis, with spontaneous recanalization by the time of the urgent coronary angiogram. Only mild spasm on apical LAD was detected, and no coronary imaging tools, such as intravascular ultrasound or optical coherence tomography, were thought to be useful in this case. No association could be made between the patient's excellent cardiorespiratory condition prior to PCI and the rare lifethreatening complication experienced.

Non-invasive imaging techniques such as transthoracic/ transesophageal echocardiography (TTE/TEE), computed tomography (CT), and cardiac magnetic resonance imaging (CMRI) have been employed with a favourable diagnostic yield in patients with left ventricular pseudoaneurysms. TTE/TEE are 26\% and 75\% effective in procuring a definitive diagnosis, respectively. A narrow neck and a wide apex are the hallmarks of a pseudoaneurysm on echocardiography. CMRI demonstrates capability to distinguish between true and pseudoaneurysms, that too with $100 \%$ sensitivity [5]. In $50 \%$ cases are diagnosed by chest radiography. Chest x-rays of more than $50 \%$ patients may display the appearance of a mass. This proffers a clue towards the diagnosis of left ventricular pseudoaneurysm. Majority patients were reported to display non-specific ECG changes whereas 20\% displayed ST-segment elevations [13]. Inayat et al. [5] reported their case to be rare as there were no abnormal changes on the ECG. Similarly, in our case no ECG abnormalities were detected. Also, a murmur was not heard in our patient.

Left ventriculogram is one of the most conclusive diagnostic tools for this cardiac phenomenon. Contrast ventriculography persists as the gold standard however, it is scarcely used [5] Despite consideration as a part of cardiac catheterization, its use is dependent geography, region, institutional, and the individual. Individuals who oppose this imaging modality argue about cost, risk, greater amount of contrast needed, and radiation exposure. These critics agree it should be replaced with a non-invasive imaging modality. However, left ventriculography elicits useful information such as (i) ejection fraction of left ventricle; (ii) wall motion and ventricular thrombus; (iii) ventricular volume; (iv) regurgitant lesions; and (v) aneurysm, pseudoaneurysm and ventricular septal defects [13].

Management of left ventricular pseudoaneurysms includes either medical therapy or surgery. Medical therapy in asymptomatic patients with small sized $(<3 \mathrm{~cm})$ out-pouchings has been recommended. The objective of this approach is to decrease pseudoaneurysm enlargement whilst attempting to decrease ventricular wall stress by decreasing afterload and decreasing the risk of thromboembolism. In such patients regular monitoring of the left ventricular pseudoaneurysm dimensions remains critical. Inayat et al. [5] initiated angiotensin-converting enzyme inhibitors for afterload reduction and $\beta$-blockers as an anti-anginal medication for their patient. Díaz-Navarro et al. [14] optimizing the patient's heart failure prescribed cardio-selective beta-blocker agents, angiotensin-converting enzyme inhibitors, spironolactone and chronic anticoagulation therapy due to increased risk of ischemic stroke in their patient.

Surgery has been suggested for patients with giant pseudoaneurysms holding considerable risk of rupture. Primary repair may be risky as pressure exertion on the left ventricle may cause arrhythmia. In acute cases, closure of the freshly necrotic myocardium with synthetic or pericardial patches is effective. When the defect is large or located near the base of the heart, a patch may be preferable to avoid excessive traction on the myocardium. In these cases, reconstructing the left ventricle with a patch is preferable, to avoid distortion of the mitral valve apparatus or excessive traction on the ventricular edges. In cases requiring mitral valve surgery, the neck of the left ventricular pseudoaneurysm can be closed through the left atrium using an autologous pericardial patch [5]. Recently, novel percutaneous approaches have been used for smaller pseudoaneurysms with less chances of rupture, thromboembolism and cardiac failure. Septal occlusion devices can close the pseudoaneurysm percutaneously. Patch closure or primary closure should be the first option, especially in patients with symptomatic and acute pseudoaneurysms [15].

A case series reported by Yeo et al. [6] related site of a pseudoaneurysm to etiology. The most common site for the development of a pseudoaneurysm was inferior or posterolateral wall after myocardial infarction in $82 \%$ of the patients. In $87 \%$ of the patients undergoing congenital heart surgery, right ventricular outflow tract pseudoaneurysm was seen. All patients undergoing mitral valve replacement and aortic valve replacement had the disease in the posterior subannular region of the mitral valve and the subaortic region, respectively. Our patient was diagnosed with a left ventricular pseudoaneurysm and hence we could not validate their findings with our case.

Left ventricular pseudoaneurysms are prone to spontaneous rupture regardless of age or size. Hence, prompt diagnosis is of paramount importance in the management and prognosis of patients with left ventricular pseudoaneurysms. Future research should aim to stratify the risks and indications of surgery in patients with left ventricular pseudoaneurysm.

\section{Conclusion}

In conclusion, we report a rare case of left ventricular apical pseudoaneurysm formation and rupture, as an unforeseen complication after successful and non-complicated elective PCI. After exclusion of LAD acute occlusion during or after PCI, the causes for this complication remain unclear, and myocardial reperfusion injury or undetected acute stent thrombosis are suggested as the main etiologies. Fortunately, the complication was properly treated, and the patient presented great clinical outcome after one year. Many questions are yet to be investigated regarding ischemia, blood flow and reperfusion myocardial injury in the coronary system. 


\section{Acknowledgement}

None.

\section{Conflict of Interest}

The authors declare no conflict of interest.

\section{References}

1. Jneid H, Addison D, Bhatt DL, Fonarow GC, Gokak S, et al. (2017) 2017 AHA/ACC Clinical Performance and Quality Measures for Adults With ST-Elevation and Non-ST-Elevation Myocardial Infarction: A Report of the American College of Cardiology/American Heart Association Task Force on Performance Measures. J Am Coll Cardiol 70(16): 2048-2090.

2. Ibanez B, James S, Agewall S, Antunes MJ, Bucciarelli-Ducci C, et al. (2018) 2017 ESC Guidelines for the management of acute myocardial infarction in patients presenting with ST-segment elevation: The Task Force for the management of acute myocardial infarction in patients presenting with ST-segment elevation of the European Society of Cardiology (ESC). Eur Heart J 39(2): 119-177.

3. Bisoyi S, Dash AK, Nayak D, Sahoo S, Mohapatra R (2016) Left ventricular pseudoaneurysm versus aneurysm a diagnosis dilemma. Ann Card Anaesth 19(1): 169-172.

4. Fok M, Bashir M, Hammoud I, Harrington D, Kuduvalli M, et al. (2014) An apical left ventricular aneurysm rupture presenting as left breast mass 11 years after surgical repair. Ann R Coll Surg Engl 96(7): e6-7.

5. Inayat F, Ghani AR, Riaz I, Ali NS, Sarwar U, et al. (2018) Left Ventricular Pseudoaneurysm: An Overview of Diagnosis and Management. J Investig Med High Impact Case Rep 6: 2324709618792025.

6. Yeo TC, Malouf JF, Oh JK, Seward JB (1998) Clinical profile and outcome in 52 patients with cardiac pseudoaneurysm. Ann Intern Med 128(4): 299-305.
7. Frohlich GM, Meier P, White SK, Yellon DM, Hausenloy DJ (2013) Myocardial reperfusion injury: Looking beyond primary PCI. Eur Heart J 34(23): 1714-1722.

8. Yellon DM, Hausenloy DJ (2007) Myocardial reperfusion injury. The New England journal of medicine 357(11):1121-1135.

9. Zhu CG, Guo YL, Wu NQ, Tang YT, Jiang LX, et al. (2012) Very Rapid Spontaneous Recanalization of Macrodissected Right Coronary Artery Following Percutaneous Coronary Intervention Associated with Total Coronary Occlusion. J Clin Case Rep 2(7).

10. Bainey KR, Fu Y, Wagner GS, Goodman SG, Ross A, et al. (2008) Spontaneous reperfusion in ST-elevation myocardial infarction: comparison of angiographic and electrocardiographic assessments. Am Heart J 156(2):248-255.

11. Stone GW, Cox D, Garcia E, Brodie BR, Morice MC, et al. (2001) Normal flow (TIMI-3) before mechanical reperfusion therapy is an independent determinant of survival in acute myocardial infarction: analysis from the primary angioplasty in myocardial infarction trials. Circulation 104(6): 636-641.

12. Fefer P, Beigel R, Atar S, Aronson D, Pollak A, et al. (2017) Outcomes of patients presenting with clinical indices of spontaneous reperfusion in ST-elevation acute coronary syndrome undergoing deferred angiography. J Am Heart Assoc 6(7): e004552.

13. Ho HH, Sinaga DA, Lee E, Watson TJ, Fatt Hon JK (2017) Left ventricular pseudoaneurysm. J Geriatr Cardiol 14(1): 78-80.

14. Diaz-Navarro R, Nihoyannopoulos P (2017) Post-myocardial infarction left ventricular pseudoaneurysm diagnosed incidentally by echocardiography. Echo Res Pract 4(4): K37-K40.

15. Prifti E, Bonacchi M, Baboci A, Giunti G, Veshti A, et al. (2017) Surgical treatment of post-infarction left ventricular pseudoaneurysm: Case series highlighting various surgical strategies. Ann Med Surg (Lond) 16: 44-51. 